\title{
VERTICES CONTAINED IN ALL OR IN NO MINIMUM SEMITOTAL DOMINATING SET OF A TREE
}

\author{
Michael A. Henning ${ }^{1}$ \\ AND \\ Alister J. MARCON ${ }^{2}$ \\ Department of Pure and Applied Mathematics \\ University of Johannesburg \\ Auckland Park, 2006, South Africa \\ e-mail: mahenning@uj.ac.za \\ alister.marcon@gmail.com
}

\begin{abstract}
Let $G$ be a graph with no isolated vertex. In this paper, we study a parameter that is squeezed between arguably the two most important domination parameters; namely, the domination number, $\gamma(G)$, and the total domination number, $\gamma_{t}(G)$. A set $S$ of vertices in a graph $G$ is a semitotal dominating set of $G$ if it is a dominating set of $G$ and every vertex in $S$ is within distance 2 of another vertex of $S$. The semitotal domination number, $\gamma_{t 2}(G)$, is the minimum cardinality of a semitotal dominating set of $G$. We observe that $\gamma(G) \leq \gamma_{t 2}(G) \leq \gamma_{t}(G)$. We characterize the set of vertices that are contained in all, or in no minimum semitotal dominating set of a tree.
\end{abstract}

Keywords: domination, semitotal domination, trees.

2010 Mathematics Subject Classification: 05C69.

\section{REFERENCES}

[1] M. Blidia, M. Chellali and S. Khelifi, Vertices belonging to all or no minimum double dominating sets in trees, AKCE Int. J. Graphs. Comb. 2 (2005) 1-9.

\footnotetext{
${ }^{1}$ Research supported in part by the South African National Research Foundation and the University of Johannesburg.

${ }^{2}$ Funded by the South African National Research Foundation.
} 
[2] E.J. Cockayne, M.A. Henning and C.M. Mynhardt, Vertices contained in all or in no minimum total dominating set of a tree, Discrete Math. 260 (2003) 37-44. doi:10.1016/S0012-365X(02)00447-8

[3] W. Goddard, M.A. Henning and C.A. McPillan, Semitotal domination in graphs, Util. Math. 94 (2014) 67-81.

[4] T.W. Haynes, S.T. Hedetniemi and P.J. Slater, Fundamentals of Domination in Graphs (Marcel Dekker, Inc. New York, 1998).

[5] M.A. Henning, Recent results on total domination in graphs: A survey, Discrete Math. 309 (2009) 32-63. doi:10.1016/j.disc.2007.12.044

[6] M.A. Henning and A.J. Marcon, On matching and semitotal domination in graphs, Discrete Math. 324 (2014) 13-18. doi:10.1016/j.disc.2014.01.021

[7] M.A. Henning and A.J. Marcon, Semitotal domination in graphs: Partition and algorithmic results, Util. Math., to appear.

[8] M.A. Henning and M.D. Plummer, Vertices contained in all or in no minimum paired-dominating set of a tree, J. Comb. Optim. 10 (2005) 283-294. doi:10.1007/s10878-005-4107-3

[9] M.A. Henning and A. Yeo, Total domination in graphs (Springer Monographs in Mathematics, 2013).

[10] C.M. Mynhardt, Vertices contained in every minimum dominating set of a tree, J. Graph Theory 31 (1999) 163-177. doi:10.1002/(SICI)1097-0118(199907)31:3〈163::AID-JGT2〉3.0.CO;2-T

\section{APPENDIX}

We now present an example to illustrate Theorem ??. Applying our pruning process discussed in Section ?? to the rooted tree $T$ with root $v$ illustrated in Figure 1(a), we proceed as follows.

- The branch vertices $b_{3}$ and $b_{4}$ are both at maximum distance 3 from $v$ in $T$. We select $b_{3}$, where $\left|L^{3}\left(b_{3}\right)\right|=1$. Thus, $b_{3}$ is a type-(T.1) branch vertex and we delete $D\left(b_{3}\right)$ and attach a path of length 3 to $b_{3}$.

- The branch vertex at maximum distance from $v$ in the resulting tree (illustrated in Figure $1(\mathrm{~b}))$ is the vertex $b_{4}$. Since $\left|L^{1}\left(b_{4}\right)\right|>2$ and every leaf-descendant of $b_{4}$ belongs to $L^{1}\left(b_{4}\right)$, the vertex $b_{4}$ is therefore a type-(T.3) branch vertex and we delete $D\left(b_{4}\right)$ and attach a path of length 1 to $b_{4}$.

- The branch vertex at maximum distance from $v$ in the resulting tree (illustrated in Figure $1(\mathrm{c}))$ is the vertex $b_{2}$. Since $\left|L^{4}\left(b_{2}\right)\right|=1$ and $L^{1}\left(b_{2}\right)=L^{3}\left(b_{2}\right)=\emptyset$, the vertex $b_{2}$ is a type-(T.4) branch vertex and we delete $D\left(b_{2}\right)$ and attach a path of length 4 to $b_{2}$.

- The branch vertex at maximum distance from $v$ in the resulting tree (illustrated in Figure $1(\mathrm{~d}))$ is the vertex $b_{1}$. Since $\left|L^{3}\left(b_{1}\right)\right|=1$, the vertex $b_{1}$ is a type-(T.1) branch 

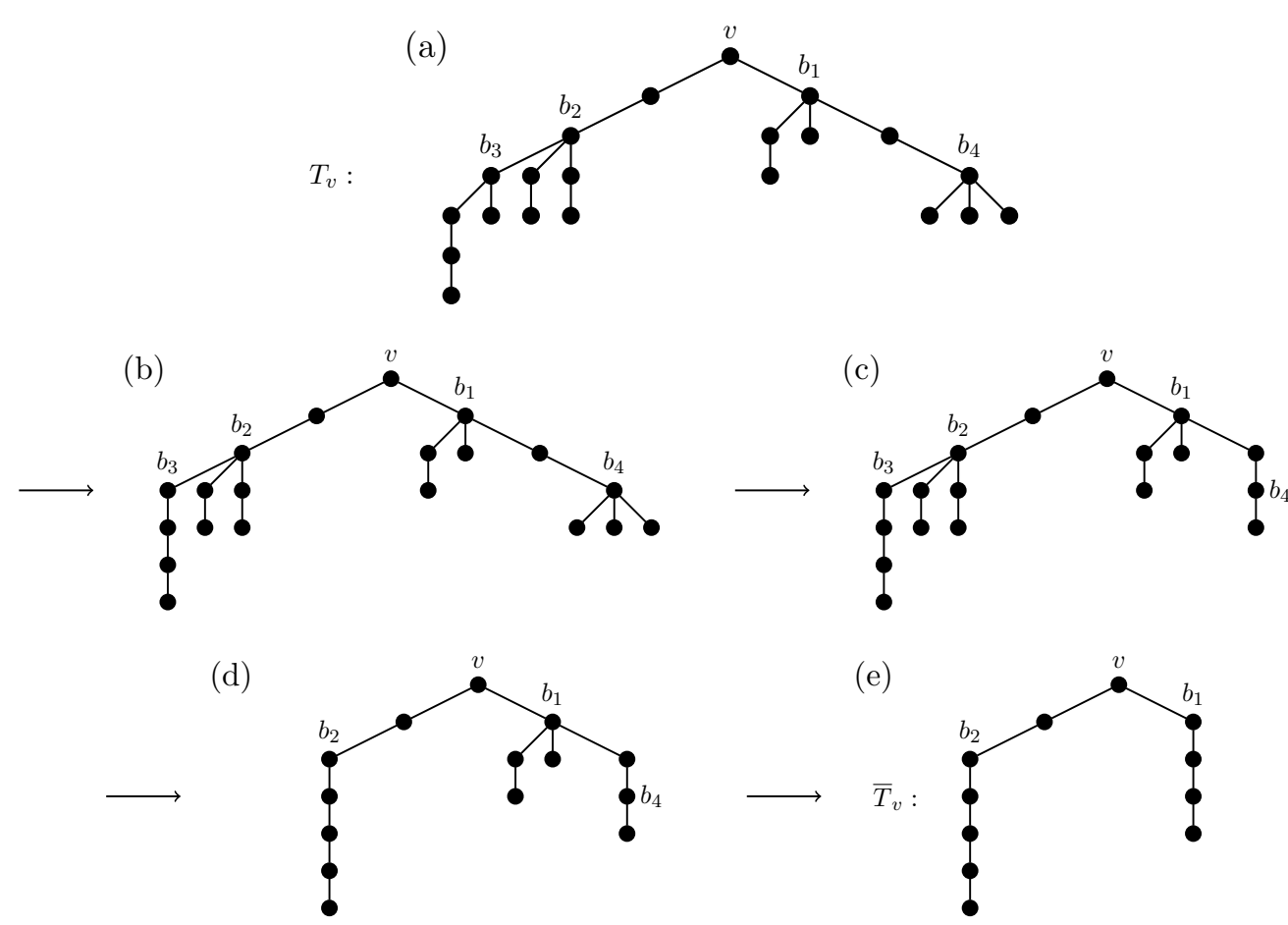

Figure 1.The pruning of a tree rooted at $v$.

vertex and we delete $D\left(b_{1}\right)$ and attach a path of length 3 to $b_{1}$. The resulting pruned tree $\bar{T}_{v}$ is illustrated in Figure $1(\mathrm{e})$.

- Since $\left|\bar{L}^{1}(v)\right|=1$ and $\left|\bar{L}^{4}(v)\right|=1$, by Theorem ??, we deduce that $v \notin \mathcal{A}_{t 2}(T) \cup$ $\mathcal{N}_{t 2}(T)$.

Received 18 December 2014

Revised 13 April 2015

Accepted 13 April 2015 